\title{
Computation of turbulent flow past an array of cylinders using a spectral method with Brinkman penalization
}

\author{
N. K.-R. Kevlahan ${ }^{1}$ and J.-M. Ghidaglia ${ }^{2}$ \\ ${ }^{1}$ Department of Mathematics and Statistics, McMaster University, Hamilton \\ L8S 4K1, Canada. ${ }^{2}$ CMLA, ENS-Cachan et CNRS UMR 8536, 61 Avenue du \\ Président Wilson, 94235 Cachan cedex, France
}

\begin{abstract}
A major difficulty in computing engineering flows at high Reynolds number is the need for non-uniform grids adapted to solid boundaries that may be moving or changing shape. These non-uniform grids are expensive to calculate and cannot be used with the most accurate or efficient numerical schemes. We present one solution to this problem: a Brinkman (volume) penalization of the obstacle which allows an efficient pseudo-spectral method to be used to solve the Navier-Stokes equations on a Cartesian grid. Although this is the most severe test of the penalization (due to the global support of the Fourier basis), it is shown that the method still yields reasonable results. We also present an analytical solution of Stokes flow calculated using the penalization which illustrates the error and continuity properties of the approach. Work is currently underway to implement the penalization approach in a wavelet basis.
\end{abstract}

\section{Introduction}

The computation of moderate Reynolds number flow without subgrid-scale models is possible for simple geometries (such as periodic boundary conditions or channel flow) due to the implementation of highly efficient and accurate spectral methods. These simulations are useful to investigate the fundamental physics of turbulence, but they are not able to calculate flows of engineering interest. For such flows (which may include complex geometries and moving or deforming solid boundaries) spectral methods cannot be used since the calculational grid must follow the shape of the obstacle (to implement the no-slip boundary condition) and should be refined near the solid boundaries to resolve the boundary layer (whose thickness $\delta$ decreases like $R e^{1 / 2}$ ). Such unstructured grids can only be used with less efficient and less accurate methods, such as finite elements. In addition, the cost of recalculating the grid to follow the movement and deformation of the obstacle is usually prohibitive.

An alternative approach to calculating flow around complex geometries is to retain the simple grid and accurate numerical method and instead change the equation. This is the essence of the 'penalization' (or fictitious domain) methods 
where an extra term is added to the Navier-Stokes equations which 'penalizes' flow in the solid region (volume penalization) or at the solid boundary (surface penalization). Such a method (called the 'immersed boundary approach') was first used by Peskin [1] to model flow around heart valves, and in modified form by Goldstein et al. to model flow two-dimensional flow around a cylinder [2] and by Goldstein \& Tuan to calculate three-dimensional channel flow over a ribbed surface [3]. Saiki \& Biringen [4] used Goldstein's method to investigate the transition to turbulence of flow past a sphere in a boundary layer. In this approach the presence of the solid boundary is simulated by adding an appropriate force field to the flow. The penalization approach appeared promising, but the precise form of the penalization was always ad hoc, often based on a mechanical model to add a body force to cancel the flow at the solid boundary. Because of the the ad hoc nature of the penalization, it was difficult to estimate the error of the method theoretically. In addition, the penalization usually involved an iterative scheme that is conceptually complicated (although relatively straightforward to implement numerically). For example, Goldstein's method required solving an iterative feedback scheme to enforce the no-slip boundary condition. Such iterative schemes make mathematical analysis of the method difficult.

Other indirect ways of enforcing boundary conditions include: adding a force at the boundary at each time step such that velocity is neutralized to second-order [5], and interpolating the velocity at the boundary using Lagrange polynomials that satisfy the no-slip condition [6].

A new penalization has recently been introduced by Angot et al. [7] following Arquis \& Caltagirone[8] which is based on the Brinkman law for flow in a porous medium. Essentially, one models the flow as a porous medium, where the permeability is infinite in the fluid part and tends to zero in the solid part. The porosity is maintained constant in the solid. The permeability $K$ of the 'solid' is then controlled by a small parameter $\epsilon: K \propto 1 / \epsilon \gg 1$. The main advantage of this method is that Angot et al. [7] have rigorously shown using asymptotic analysis that the solution of the penalized equations $\boldsymbol{u}_{\epsilon}$ converges to the solution of the Navier-Stokes equations with the correct boundary conditions $\boldsymbol{u}$ as $\epsilon \rightarrow 0$ with a well-controlled global error of $O\left(\epsilon^{3 / 4}\right)$. This means that the penalization can be used with confidence, and the error can be chosen as small as desired, merely by using an appropriate value for $\epsilon$. This rigour (and the fact that it is a volume rather than a surface penalization) distinguishes the Brinkman penalization from other penalization methods. The penalization term is also extremely simple: one adds a term which is $-1 / \epsilon \boldsymbol{u}$ in the solid and zero elsewhere.

In this paper our goal is to make a first step towards the use of spectral (and eventually wavelet) methods for computing the solution of complex flows. More precisely, we would like to show that using the Brinkman penalization method it is possible to perform stable and accurate computations of incompressible viscous flow past an arbitrary obstacle using a classical pseudo-spectral method 
based on a Fourier decomposition. Because of its global support, the Fourier basis is not well-adapted to non-smooth functions (e.g. discontinuous functions produce Gibbs oscillations) and thus if the penalization produces reasonable results with the pseudo-spectral method it should work well for any other method (e.g. a wavelet method with whose basis functions have compact support). Apart from its exponential convergence properties (for smooth $C^{\infty}$ functions), the pseudo-spectral method is interesting because of its ease of implementation in two or three dimensions and its applicability to problems with periodic boundary conditions.

The paper is organized as follows. $§ 2$ presents the theory of the penalization and an analytic solution of Stokes flow. This calculation illustrates the error and smoothness of the penalization solution near a solid boundary. Then in $\S 3$ we describe how the penalization is implemented in a two-dimensional pseudospectral numerical code. Some results of applying a spectral and second-order finite difference code to the Stokes flow problem are also presented. The results of applying the penalized code to a selection of test problems for cylinder arrays are presented in $\S 4$. These problems are relevant to flow past tube bundles in the heat exchangers of steam generators. Finally, the advantages and disadvantages of the method are discussed and perspectives for future research are suggested in $\S 5$.

\section{Theory}

\subsection{Brinkman penalization}

Let us consider a viscous incompressible fluid governed by the Navier-Stokes equations

$$
\begin{aligned}
\frac{\partial \boldsymbol{u}}{\partial t}+\boldsymbol{u} \cdot \nabla \boldsymbol{u}+\nabla P & =\nu \Delta \boldsymbol{u} \\
\nabla \cdot \boldsymbol{u} & =0 .
\end{aligned}
$$

We focus here on the case where the fluid occupies the complement in the plane $R^{2}$ of a periodic lattice of obstacles $O_{i}$ (see figure 1 ). The boundary conditions associated with this problem are therefore:

$$
\begin{array}{r}
\boldsymbol{u} \text { is } Q \text {-periodic, } Q=] 0, L_{1}[\times] 0, L_{2}[, \\
\boldsymbol{u}=0 \text { on } \partial O_{i}, \forall i
\end{array}
$$

When a Fourier Galerkin method is used to compute the solution of (1) - (4), the solution is decomposed as

$$
\boldsymbol{u}\left(x_{1}, x_{2}, t\right)=\sum_{k \in Z^{2}} \boldsymbol{u}_{k}(t) \exp \left[i 2 \pi\left(\frac{k_{1} x_{1}}{L_{1}}+\frac{k_{2} x_{2}}{L_{2}}\right)\right]
$$




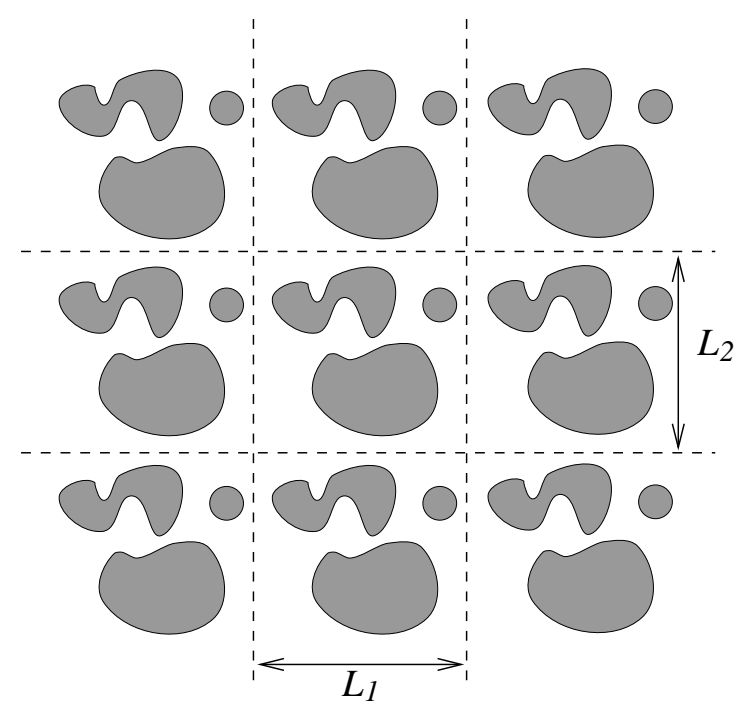

Figure 1: Periodic lattice of obstacles

and the obstacles are defined by the boundary condition (4), which is impossible to implement for arbitrary obstacles $O_{i}$.

In order to use the Fourier decomposition (5) for arbitrary obstacles we replace (1) - (4) by the following set of penalized equations

$$
\begin{aligned}
\frac{\partial \boldsymbol{u}_{\epsilon}}{\partial t}+\boldsymbol{u}_{\epsilon} \cdot \nabla \boldsymbol{u}_{\epsilon}+\nabla P_{\epsilon} & =\nu \Delta \boldsymbol{u}_{\epsilon}-\frac{1}{\epsilon} \chi_{0} \boldsymbol{u}_{\epsilon}, \\
\nabla \cdot \boldsymbol{u}_{\epsilon} & =0
\end{aligned}
$$

$$
\boldsymbol{u}_{\epsilon} \text { is } Q \text {-periodic, }
$$

and now (6) and (7) are to be satisfied in the whole plane $R^{2}$. Here $\epsilon>0$ is a penalization coefficient and $\chi_{0}$ denotes the characteristic (or mask) function

$$
\chi_{0}(\boldsymbol{x}, t)= \begin{cases}1 & \text { if } \boldsymbol{x} \in O_{i}, \\ 0 & \text { otherwise }\end{cases}
$$

As $\epsilon \rightarrow 0$, it was proved theoretically by Angot, Bruneau \& Fabrie [7] that the solutions of the penalized equations (6) to (8) converge to that of the NavierStokes equations with the correct boundary conditions (1) - (4). More precisely, the upper bound on the global error of the penalization for steady flow was shown to be $[7]$

$$
\left\|\boldsymbol{u}-\boldsymbol{u}_{\epsilon}\right\| \leq C \epsilon^{1 / 4} .
$$


This penalization has been implemented in a finite difference code by Khadra et al.[9] for flow around a cylinder and was found to give very good results. In fact, the actual error was slightly better, $O(\epsilon)$. It is important to note that $\epsilon$ is an arbitrary parameter, independent of the spatial or temporal discretization, and thus the boundary conditions can be enforced to any desired accuracy by choosing $\epsilon$ appropriately. This property distinguishes the Brinkman method from other penalization schemes and allows the error to be controlled precisely.

Another advantage of the Brinkman penalization is that the force $\boldsymbol{F}_{i}$ acting on an obstacle $O_{i}$ can be found by simply integrating the penalization term over the volume of the obstacle (see [10] and [11] for details):

$$
\boldsymbol{F}_{i}=\frac{1}{\epsilon} \int_{O_{i}} \boldsymbol{u} \mathrm{d} \boldsymbol{x} .
$$

Thus, the calculation of lift and drag on an obstacle can be made simply, accurately and at very low cost. (This result can be found by integrating the penalized Navier-Stokes equations and noting that $\boldsymbol{u}$ and $\boldsymbol{u} \cdot \nabla \boldsymbol{u}$ are negligible in the solid part.)

\subsection{Penalization in two dimensions}

Note that the penalization applies equally to one, two or three dimensions. In this paper, however, we focus on two-dimensional flow, and hence we would like to use the efficient vorticity-stream function $(\omega-\psi)$ form of the NavierStokes equations. We have chosen this formulation since it is more efficient than the primitive variables formulation in two dimensions, and is thus the standard choice for two-dimensional turbulence simulations. It is also a tough test for the penalization approach since the penalization term becomes the curl of a Heaviside function (which is singular). If the method gives reasonable results in this case, it should work even better for the primitive variable formulation in two and three dimensions. In addition, we plan to implement the penalization in an adaptive wavelet code (which relies on a large compression of the vorticity field), so we need to ensure the method works on the vorticity equation.

The penalized form of the vorticity equation is

$$
\begin{aligned}
\frac{\partial \omega}{\partial t}+J(\psi, \omega) & =\nu \Delta \omega-\frac{1}{\epsilon} \operatorname{curl}\left(\chi_{0} \underline{\operatorname{curl}} \psi\right) \\
-\Delta \psi & =\omega
\end{aligned}
$$

where we have set

$$
\begin{aligned}
& J(\psi, \omega) \equiv \frac{\partial \psi}{\partial x_{1}} \frac{\partial \omega}{\partial x_{2}}-\frac{\partial \psi}{\partial x_{2}} \frac{\partial \omega}{\partial x_{1}} \\
& \underline{\operatorname{curl}} \psi \equiv\left(\frac{\partial \psi}{\partial x_{2}},-\frac{\partial \psi}{\partial x_{1}}\right), \\
& \operatorname{curl} \boldsymbol{v} \equiv\left(\frac{\partial \boldsymbol{v}}{\partial x_{2}}-\frac{\partial \boldsymbol{v}}{\partial x_{1}}\right) .
\end{aligned}
$$


Of course if $(\psi, \omega)$ solves (12) - (13) then $\boldsymbol{u}=\underline{\text { curl }} \psi$ solves (1)-(4) for a suitable pressure function $P_{\epsilon}$.

This formulation is preferred for at least two reasons. First, the incompressibility equation (2) is automatically satisfied, and we do not need to compute the pressure. Secondly, in the context of two-dimensional flows, the vorticity is the physically relevant variable whose size and shape are directly related to the physical phenomenon involved (instabilities, drag, lift etc.).

We have limited our investigations here to the Fourier decomposition and two-dimensional flows. Extensions to (i) wavelet decomposition, and (ii) threedimensional flow are currently under way and will be reported elsewhere (see also $\S 5$ of this paper).

\subsection{Application to Stokes flow}

To help understand what the penalized approximation to the solution of the Navier-Stokes equations looks like near a solid boundary we consider the simple case of Stokes flow. Stokes solution describes a uni-directional flow accelerated impulsively from rest and bounded by an infinite flat plate parallel to the flow. The velocity of the flow must be zero at the surface of the plate, and thus the flow develops a typical 'boundary layer' shear profile that progressively diffuses vorticity into the interior of the flow. In this case the Navier-Stokes equations become

$$
\frac{\partial u}{\partial t}=\nu \frac{\partial^{2} u}{\partial x_{2}^{2}}
$$

where $u=u_{1}\left(x_{2}, t\right), u\left(x_{2}, 0\right)=1, u(0, t)=0$ and $x_{2} \geq 0$ (note that we consider the lower half-plane to be solid). It is assumed that the flow remains unidirectional (i.e. that no instabilities develop). The above equations can be easily solved, giving

$$
u\left(x_{2}, t\right)=\operatorname{erf}\left(\frac{x_{2}}{\sqrt{4 \nu t}}\right)
$$

where erf is the usual error function: $\operatorname{erf}(z) \equiv \frac{2}{\sqrt{\pi}} \int_{0}^{z} e^{-s^{2}} d s$.

The force on the wall is given by

$$
\left.\left(\frac{\partial u}{\partial x_{2}}\right)\right|_{x_{2}=0}=\left.\frac{\partial}{\partial x_{2}} \operatorname{erf}\left(\frac{x_{2}}{\sqrt{4 \nu t}}\right)\right|_{x_{2}=0}=\frac{1}{\sqrt{\pi \nu t}} .
$$

Now, the penalized approximation to Stokes flow is given by

$$
\frac{\partial u_{\epsilon}}{\partial t}=-\frac{1}{\epsilon} H\left(-x_{2}\right) u_{\epsilon}+\nu \frac{\partial^{2} u_{\epsilon}}{\partial x_{2}^{2}}
$$

where $H(x)$ is the Heaviside function, $u_{\epsilon}\left(x_{2}, 0\right)=1$ and $u_{\epsilon}(\cdot, t)$ is defined on the entire plane $R^{2}$ (see e.g. [12]). We solve the penalized equation using the 
Laplace transform,

$$
z\left(x_{2}, \alpha\right)=\int_{0}^{\infty} e^{-\alpha t} u_{\epsilon}\left(x_{2}, t\right) \mathrm{d} t .
$$

Taking the Laplace transform of (20) (letting $\nu=1 / 2$ for simplicity) we obtain the following equations,

$$
\begin{array}{ll}
\alpha z=\frac{1}{2} z^{\prime \prime}-\beta z+1, & x_{2}<0, \\
\alpha z=\frac{1}{2} z^{\prime \prime}+1, & x_{2}>0,
\end{array}
$$

with boundary conditions $z\left(0^{+}\right)=z\left(0^{-}\right), z^{\prime}\left(0^{+}\right)=z^{\prime}\left(0^{-}\right)$, where the prime indicates the derivative with respect to $x_{2}$ and $\beta=1 / \epsilon$. The solution of the above equations is

$$
\begin{array}{ll}
z=A e^{-|x| \sqrt{2(\alpha+\beta)}}+\frac{1}{\alpha+\beta}, & x<0, \\
z=B e^{-x \sqrt{2 \alpha}}+\frac{1}{\alpha}, & x>0,
\end{array}
$$

with

$$
A=\frac{\sqrt{\alpha+\beta}-\sqrt{\alpha}}{\sqrt{\alpha}(\alpha+\beta)}, B=\frac{-\sqrt{\alpha+\beta}+\sqrt{\alpha}}{\alpha \sqrt{\alpha+\beta}} .
$$

Taking the inverse Laplace transform of (23) and substituting $\beta=1 / \epsilon$ we find

$$
\begin{aligned}
& u_{\epsilon}\left(x_{2}, t\right)=e^{-t / \epsilon}\left[\operatorname{erf}\left(\frac{-x_{2}}{\sqrt{2 t}}\right)+\frac{1}{\pi} \int_{0}^{1} \frac{\exp \left(t y / \epsilon-\frac{1}{2 t} \frac{x_{2}^{2}}{1-y}\right)}{\sqrt{y} \sqrt{1-y}} \mathrm{~d} y\right], \quad x_{2}<0, \\
& u_{\epsilon}\left(x_{2}, t\right)=\quad \operatorname{erf}\left(\frac{x_{2}}{\sqrt{2 t}}\right)+\frac{1}{\pi} \int_{0}^{1} \frac{\exp \left(-t y / \epsilon-\frac{1}{2 t} \frac{x_{2}^{2}}{1-y}\right)}{\sqrt{y} \sqrt{1-y}} \mathrm{~d} y, \quad x_{2}>0 .
\end{aligned}
$$

Note that the first term on the right hand side of (25) is the exact solution $u\left(x_{2}, t\right)$, and therefore the second term is the error.

We observe that for $x_{2}>0$, i.e. in the fluid, the error satisfies

$$
0 \leq u_{\epsilon}\left(x_{2}, t\right)-u\left(x_{2}, t\right) \leq \frac{1}{\pi} \int_{0}^{1} \frac{1}{\sqrt{y} \sqrt{1-y}} \mathrm{~d} y=1 .
$$

Hence,

$$
\int_{0}^{\infty}\left|u_{\epsilon}\left(x_{2}, t\right)-u\left(x_{2}, t\right)\right|^{2} \mathrm{~d} x_{2} \leq \int_{0}^{\infty}\left|u_{\epsilon}\left(x_{2}, t\right)-u\left(x_{2}, t\right)\right| \mathrm{d} x_{2}=\sqrt{\frac{\epsilon}{2}} \operatorname{erf}\left(\sqrt{\frac{t}{\epsilon}}\right) .
$$

Moreover, integrating the above expression with respect to time, we find,

$$
\begin{aligned}
\frac{1}{T} \int_{0}^{T} \int_{0}^{\infty} \mid u_{\epsilon}\left(x_{2}, t\right) & -\left.u\left(x_{2}, t\right)\right|^{2} \mathrm{~d} x_{2} \mathrm{~d} t \leq \\
& \leq \sqrt{\frac{\epsilon}{2}}\left[\left(1-\frac{\epsilon}{2 T}\right) \operatorname{erf}\left(\sqrt{\frac{T}{\epsilon}}\right)+\sqrt{\frac{\epsilon}{\pi T}} e^{-T / \epsilon}\right]
\end{aligned}
$$


This shows that the error between the exact and the approximate solutions in the fluid is of the order of $\sqrt{\epsilon}$. Angot et al [7] (Theorem 4.2) have proved that in general this error is bounded by $C \epsilon^{1 / 4}$, which is consistent with our computations for the particular case of Stokes flow.

Concerning the asymptotic behaviour of $u_{\epsilon}$, as $\epsilon \rightarrow 0$ (as in the penalization approximation), when $x$ and $t$ are fixed, the integrals (24) and (25) can be estimated as

$$
\begin{array}{ll}
u_{\epsilon}\left(x_{2}, t\right) \leq e^{x_{2} \sqrt{2 / \epsilon}}\left(\sqrt{\frac{\epsilon}{\pi t}}+e^{-t / \epsilon}\right), & x_{2}<0 \\
u_{\epsilon}\left(x_{2}, t\right) \approx \operatorname{erf}\left(\frac{x_{2}}{\sqrt{2 t}}\right)+\sqrt{\frac{\epsilon}{\pi t}} e^{-x_{2}^{2} /(2 t)}, & x_{2}>0
\end{array}
$$

The above results can be generalized to arbitrary viscosity $\nu$, giving

$$
\begin{array}{ll}
u_{\epsilon}\left(x_{2}, t\right) \leq e^{x_{2} / \sqrt{\nu \epsilon}}\left(\sqrt{\frac{\epsilon}{\pi t}}+e^{-t / \epsilon}\right), & x_{2}<0, \\
u_{\epsilon}\left(x_{2}, t\right) \approx \operatorname{erf}\left(\frac{x_{2}}{\sqrt{4 \nu t}}\right)+\sqrt{\frac{\epsilon}{\pi t}} e^{-x_{2}^{2} /(4 \nu t)}, & x_{2}>0 .
\end{array}
$$

Equation $(29)_{1}$ shows that the velocity decreases exponentially fast inside the solid and is thus evanescent. The 'skin-depth' inside the obstacle is $O\left(\epsilon^{1 / 2}\right)$. This is consistent with the physical basis of the penalization since the solid is modelled as a porous obstacle with permeability $K \propto 1 / \epsilon$, and the thickness of the boundary layer inside a porous obstacle is $O\left(K^{1 / 2}\right)$. Thus, to numerically compute the force on an obstacle, one need only calculate the evanescent flow near its surface. This result has important implications since it means that an adaptive method (e.g. wavelet-based) need not resolve the entire volume of the obstacle, only a thin shell near its surface.

Both $u_{\epsilon}$ and $u_{\epsilon}^{\prime}$ are continuous at $x_{2}=0$, while $u_{\epsilon}^{\prime \prime}$ is discontinuous. At the boundary the velocity and its gradient take on (at leading order) the following values,

$$
u_{\epsilon}(0, t)=\sqrt{\frac{\epsilon}{\pi t}}+O(\epsilon), \quad \frac{\partial u_{\epsilon}}{\partial x}(0, t)=\frac{1}{\sqrt{\pi \nu t}}\left(1-e^{-t / \epsilon}\right) .
$$

Note that the error in the gradient at the surface of the obstacle is exponentially small. The fact that the solution has continuity $C^{1}$ at the boundary (but is smooth elsewhere) should help in choosing an appropriate numerical method.

In the penalization method the force on an obstacle is calculated by an integration over its volume. We calculate the force by integrating (20) from $-\infty$ to 0 (i.e. over the solid) which leads to:

$$
\frac{d}{d t} F_{\epsilon}+\frac{1}{\epsilon} F_{\epsilon}=\frac{1}{\epsilon} \frac{\partial u_{\epsilon}}{\partial x}(0, t)=\frac{1}{\epsilon} \frac{1}{\sqrt{\pi \nu t}}\left(1-e^{-t / \epsilon}\right) .
$$

This equation has the solution

$$
F_{\epsilon}(t)=C e^{-t / \epsilon}+e^{-t / \epsilon} \int_{0}^{t} e^{s / \epsilon} \frac{1}{\epsilon} \frac{1}{\sqrt{\pi \nu s}}\left(1-e^{-s / \epsilon}\right) \mathrm{d} s,
$$


where $C$ is a constant. Since we are interested in the limit $\epsilon \rightarrow 0$ with $\mathrm{t}$ fixed, we neglect the first term. The integral in (31) can be evaluated, giving

$$
F_{\epsilon}(t)=e^{-t / \epsilon}\left(\frac{1}{\sqrt{\epsilon \nu}} \operatorname{erfi}\left(\sqrt{\frac{t}{\epsilon}}\right)-\frac{2}{\epsilon} \sqrt{\frac{t}{\pi \nu}}\right),
$$

where $\operatorname{erfi}(x)=-i \operatorname{erf}(i x)$. Again, we neglect the second term and approximate the first term for large $t / \epsilon$ using

$$
\operatorname{erfi}(x)=\frac{e^{x^{2}}}{\sqrt{\pi} x}\left(1-\frac{1}{2} \frac{1}{x^{2}}+O\left(\frac{1}{x^{4}}\right)\right) .
$$

which gives the following expression for the penalized approximation to the force,

$$
F_{\epsilon}(t)=\frac{1}{\sqrt{\pi \nu t}}\left(1-\frac{\epsilon}{2 t}+O\left(\epsilon^{2}\right)\right) .
$$

Note that the error in the force compared with the exact result (19) is $O(\epsilon)$, i.e. much smaller than the error in the boundary condition (which is is $O\left(\epsilon^{1 / 2}\right)$ ).

Although Stokes flow is linear and uni-directional, it generates a solution to the three-dimensional Navier-Stokes equation. One might naturally ask whether the situation described above is exceptional or gives some indication of the general solution to the Navier-Stokes equations. It can be argued that in the evanescent layer of thickness $O(\sqrt{\epsilon})$ near a smooth boundary, the flow is approximately linear (since the convective terms become negligible with respect to the diffusive and penalized terms) and parallel to the boundary. Thus the results obtained above for the error and behaviour of the penalized solution should also hold for most three-dimensional flows.

An important result of this section is that even though the boundary condition is enforced to $O\left(\epsilon^{1 / 2}\right)$ by the penalization method, the error in the force on the obstacle is only $O(\epsilon)$ (see 32 ). The shear stress at the boundary is perhaps the most physically important quantity, and (30) shows that its error is exponentially small. Furthermore, the error in the velocity decreases rapidly away from the boundary (see 29). Taken together, these results suggest that overall the Brinkman penalization may be expected to give better than $O\left(\epsilon^{1 / 2}\right)$ accuracy (indeed Khadra et al. [9] found numerical evidence for $O(\epsilon)$ accuracy in two-dimensional flows). Finally, we concluded that an adaptive numerical method need only calculate the fictitious flow inside the obstacle to a skin-depth of $O\left(\epsilon^{1 / 2}\right)$.

\section{$3 \quad$ Numerical method}

\subsection{Pseudo-spectral scheme}

We use a standard pseudo-spectral method on a rectangular domain with periodic boundary conditions to discretize (12) (see, e.g., [14] for details). This 
means that derivatives are calculated in Fourier space, while products are calculated by returning to physical space. The diffusion term $\partial^{2} \omega / \partial \boldsymbol{x}^{2}$ is integrated exactly in time (by solving the diffusion equation in Fourier space), while a 2nd-order Adams-Bashforth scheme is used for the rest of the right hand side. The resulting scheme is

$$
\hat{\omega}_{n+1}=\hat{\omega}_{n} e^{-\nu k^{2} \Delta t}+\frac{3}{2} \Delta t \hat{g}_{n} e^{-\nu k^{2} \Delta t}-\frac{1}{2} \Delta t \hat{g}_{n-1} e^{-\nu k^{2} 2 \Delta t},
$$

where $\boldsymbol{k}=\left(k_{1}, k_{2}\right)$ is the wavenumber, $k=|\boldsymbol{k}|, \Delta t$ is the time step and

$$
\hat{g}(\boldsymbol{k})=-\hat{J}(\psi, \omega)+\frac{i}{\epsilon}\left(k_{1} \chi_{0} \frac{\partial \psi}{\partial x_{1}}+k_{2} \chi_{0} \frac{\partial \psi}{\partial x_{2}}\right) .
$$

Note that the first term on the right hand side is the Fourier transform of the Jacobian, while the second is the Fourier transform of the penalization. Thus the mask $\chi_{0}$ is applied to derivatives of the stream function (the velocity) in physical space and the product is then transformed back to Fourier space.

Because the Fourier series is truncated, the wavenumbers are bounded as $-N_{1} / 2 \leq k_{1} \leq N_{1} / 2=k_{1 \max }$ and $-N_{2} / 2 \leq k_{2} \leq N_{2} / 2=k_{2 \max }$ where $N_{1}$ and $N_{2}$ are the numbers of grid points in the $x_{1}$ and $x_{2}$ directions. Note that the grid is uniform. In order to avoid the creation of scales too small to be resolved on the grid (i.e. wavenumbers $n k_{\max }, n=2,3,4, \ldots$ ) by terms involving products, the vorticity is de-aliased at each time step by truncating in wavenumber space using the $2 / 3$-rule,

$$
\hat{\omega}(\boldsymbol{k}, t)=\left\{\begin{array}{lll}
0 & \text { if } & \left(\frac{k_{1}^{2}}{2 / 3 N_{1}}\right)^{2}+\left(\frac{k_{2}^{2}}{2 / 3 N_{2}}\right)^{2} \geq 1 \\
\hat{\omega}(\boldsymbol{k}, t) & \text { if } \quad\left(\frac{k_{1}^{2}}{2 / 3 N_{1}}\right)^{2}+\left(\frac{k_{2}^{2}}{2 / 3 N_{2}}\right)^{2}<1
\end{array}\right.
$$

Working in the $\omega-\psi$ formulation is computationally efficient in two dimensions. However, the vorticity is less smooth than the velocity (in fact we expect that $\left.\omega \in C^{0}\right)$. Thus, this application is an extreme test of the general applicability of the penalization method, since spectral methods are poorly adapted to functions with strong gradients (although they have been used to calculate shocks, usually with some form of high wavenumber filtering [15]).

The explicit time scheme used here has the drawback of linking the time step $\Delta t$ and the penalization parameter $\epsilon$. Since the problem is stiff and we use an explicit method, we require $\Delta t \sim \epsilon$ to ensure numerical stability. This is a drawback, although in practise we are able to use a sufficiently small value, $\epsilon \approx$ $10^{-3}$. In the following section we use a Krylov sub-space method (which allows $\epsilon$ to be chosen independently of $\Delta t$ ) to investigate the convergence properties of the pseudo-spectral method in the case of one-dimensional channel flow (similar to the case discussed in $\S 2.3$ ). 


\subsection{Analysis of pseudo-spectral method for channel flow}

In this section we investigate the numerical properties of the pseudo-spectral method for the Brinkman penalization by applying it to the one-dimensional primitive equation (20) for uni-directional channel flow. Because of the simplicity of the equation, and the availability of analytic solutions for both the penalized equations and the physical equations, this is an ideal problem for studying the convergence properties of the numerical method. So that $\epsilon$ may be varied independently of $\Delta t$ we employ a GMRES Krylov sub-space method in time (see [16] for a discussion of the Krylov method). The Krylov method has the advantage that it is stiffly stable for linear problems and the order of the method can be easily changed by changing the dimension of the Krylov subspace.

The physical problem is similar to the Stokes flow considered in $\S 2.3$, except that the flow is now bounded by two parallel walls. If the flow is assumed to remain uni-directional the velocity can be easily calculated (see [17]),

$$
u\left(x_{2}, t\right)=\frac{2 u_{0}}{\pi} \sum_{n=1}^{\infty} \frac{1}{n} \exp \left(-n^{2} \pi^{2} \frac{\nu t}{d^{2}}\right) \sin \left(\frac{n \pi x_{2}}{d}\right)\left(1-(-1)^{n}\right)
$$

where $u=u_{1}, u_{0}$ is the initial velocity, and $d$ is the width of the channel. (Note that this flow can be calculated on a periodic domain.) For short times and near the walls (while the velocity is close to $u_{0}$ in the centre of the flow) the solution of the associated penalized equations is given by (29) appropriately shifted and scaled.

Because we are using the penalized equations, rather than the Navier-Stokes equations there are in fact two types of error. The first type of error is the pure numerical error associated with solving the penalized equation numerically. The second error is the 'net' physical error which is a combination of the numerical error and the penalization error. Because we know both the exact solution of the penalized equations (given by 29 for small times) and the exact solution of the physical problem (given by 36) we can quantify both sources of error separately.

We found that the convergence of $L^{1}$ error of the pseudo-spectral method compared to the solution (36) was only linear with $\Delta x$, except for a special relation between $\epsilon$ and $\Delta x\left(\epsilon=\Delta x^{2.3}\right.$ for the pseudo-spectral method and $\epsilon=\Delta x^{3}$ for the finite difference method) which lowered the error to $O\left(\Delta x^{2}\right)$. However, the convergence of the error compared to the exact solution of the penalized equations was always linear. Although the convergence of the physical error is only linear in general (consistent with the $C^{1}$ continuity of the penalized solution) the actual error is quite small $\left(10^{-3}\right.$ for 128 points), and there is no sign of Gibbs oscillations.

A second-order finite difference method was also applied to the same problem, and showed similar convergence properties. The main difference was that 


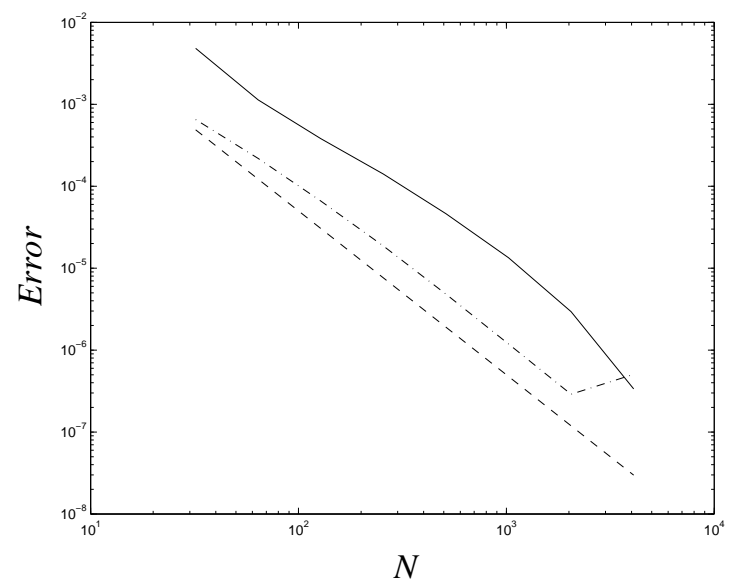

Figure 2: Error as a function of number of grid-points $N$ for optimum choice of $\epsilon=\Delta x^{p}$. - pseudo-spectral, $-\cdot-2$ nd-order finite difference, $-\cdots N^{-2}$.

the actual physical error was approximately twice as small as for the pseudospectral method. The error results for spectral and finite differences are shown in figure 2 for the optimal relation between $\Delta x$ and $\epsilon$.

These results suggest that in general the overall error of the pseudo-spectral method should be between linear and quadratic, and that Gibbs oscillations should not be important. Of course, in two dimensions we use the $\omega-\psi$ formulation which might be expected to have some Gibbs oscillations and a slightly higher error (since vorticity is less smooth than velocity). The nonlinear term might make some difference, although it should not be important in the boundary layer near the surface of the obstacle. In the following section the numerical results for the two-dimensional simulation are compared with results from laboratory experiments.

\section{Results}

\subsection{Widely separated cylinders}

In order to check that the pseudo-spectral method applied to the Brinkman penalization gives reasonable results we first apply it to the case of widely separated cylinders in long rectangular domains. This configuration was chosen because it approximates an isolated cylinder for moderate times (until shed vortices exit and re-enter the domain). The two-dimensional flow past a circular cylinder has also been calculated recently using a formally second-order accurate immersed 
boundary technique by Lai \& Peskin [13]. The resolution used for the simulation is $512 \times 128$ grid points and the physical dimensions are $20 \times 5$ cylinder diameters. The transition to vortex shedding at $R e=200$ (based on cylinder diameter and mean flow velocity) is shown in figure 3 . The simulation shows clearly the growth of the instability and the establishment of a regular vortex shedding regime. Note that some Gibbs oscillations are present around the cylinder, but these are small in amplitude and do not grow or perturb the rest of the flow. It appears that the oscillations are benign, as was observed by Goldstein et al.[2] who used a different penalization method with a pseudo-spectral scheme to calculate flow around a cylinder. The Strouhal vortex shedding frequency calculated from the oscillating lift force is $S t=f D / U_{\infty}=0.218$, which is within $10 \%$ of the value of $S t=0.195$ found by Williamson [18] for a truly isolated cylinder.

The Gibbs oscillations, although benign, are unphysical and it would be better to eliminate them. To do this we tried smoothing the edge of the mask $\chi_{0}$ over seven grid points. The smoothed cylinder generated much smaller oscillations, but also had increased drag and lift (and a slightly smaller Strouhal number) than the non-smoothed case, see figure 4 . This suggests that smoothing the edge of the cylinder changes the physics of the flow-obstacle interaction significantly. We are, in effect, calculating the flow around a slightly 'fuzzy' cylinder (a tennis ball?). In any case, we will see in the following section that at higher Reynolds numbers the Gibbs oscillations become completely negligible.

In this section we have seen that the combination of Brinkman penalization with the pseudo-spectral method gives reasonable results for the well-understood case of an isolated cylinder. In the following section we investigate the industrially important, but more complicated case of closely spaced periodic arrays of cylinders.

\subsection{Closely spaced square cylinder arrays}

The case of flow through closely spaced periodic cylinder arrays is important for many industrial applications, e.g. flow around nuclear fuel rods, flow past heat exchange coils in steam generators. At moderate to high Reynolds numbers the flow past cylinder arrays can generate strong oscillating forces that can destroy a heat exchanger if these forces are close to the resonant frequency of the tubes. It is thus important to understand how such forces arise, and how they vary in different tube configurations. One important question is how the flow regime changes as a function of the angle of incidence of the mean flow with respect to the axis of the tube array. Because of its periodic boundary conditions, the pseudo-spectral method is ideally suited to investigating flow in tube bundles. Although this flow is important industrially, it has received little attention from the fluid dynamics community.

We consider only the simplest case of a single fixed cylinder per period in two-dimensional flow. The case of elastic cylinders is under investigation, and is 


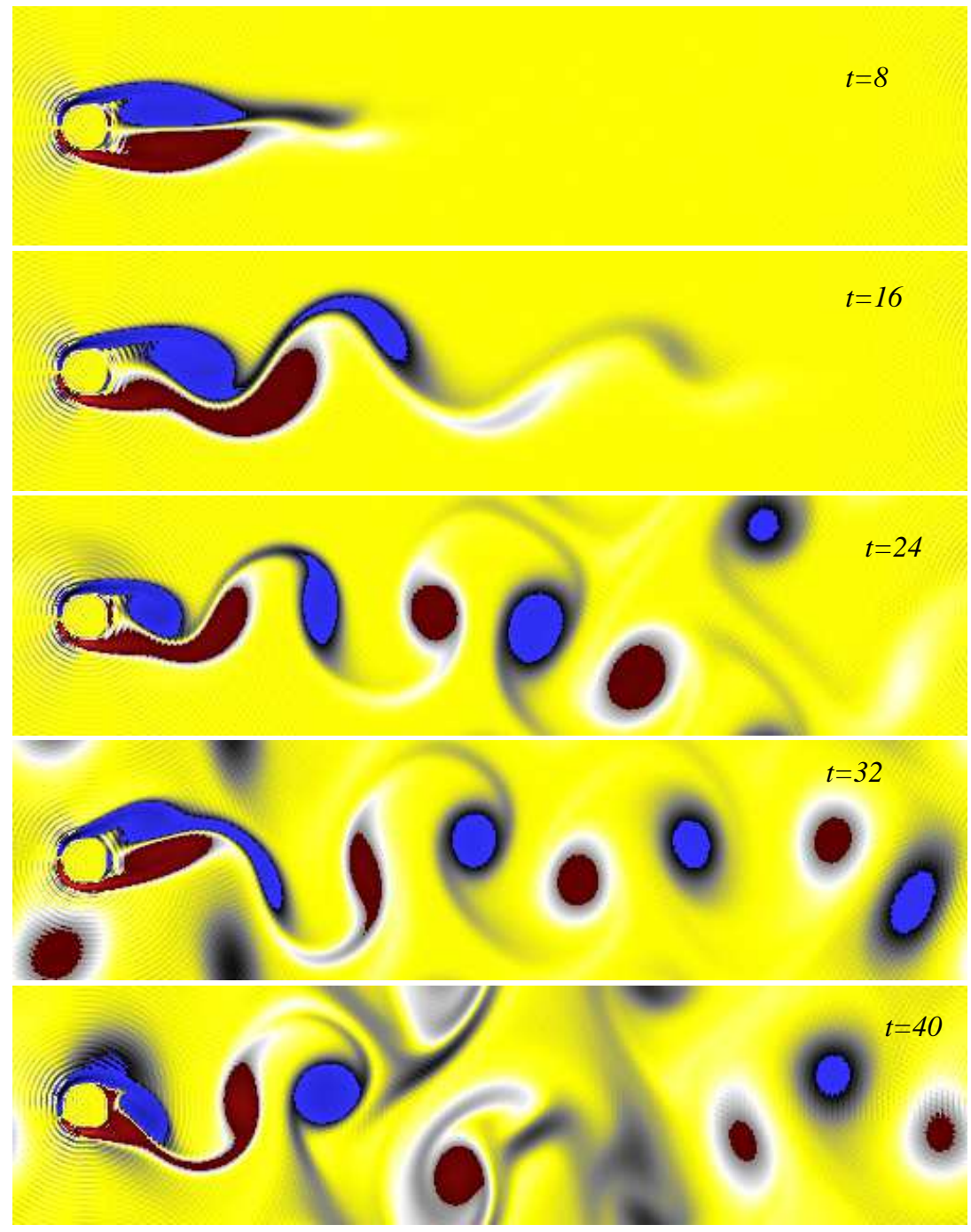

$R e=200 \quad 20 \times 5(512 \times 128)$ without smoothing

Figure 3: Development of vortex shedding around a single cylinder at $R e=200$. Resolution is $512 \times 128$ and positive vorticity is red, negative vorticity is blue. Note that some Gibbs oscillations are present, but they don't appear to grow in time or perturb the flow. 

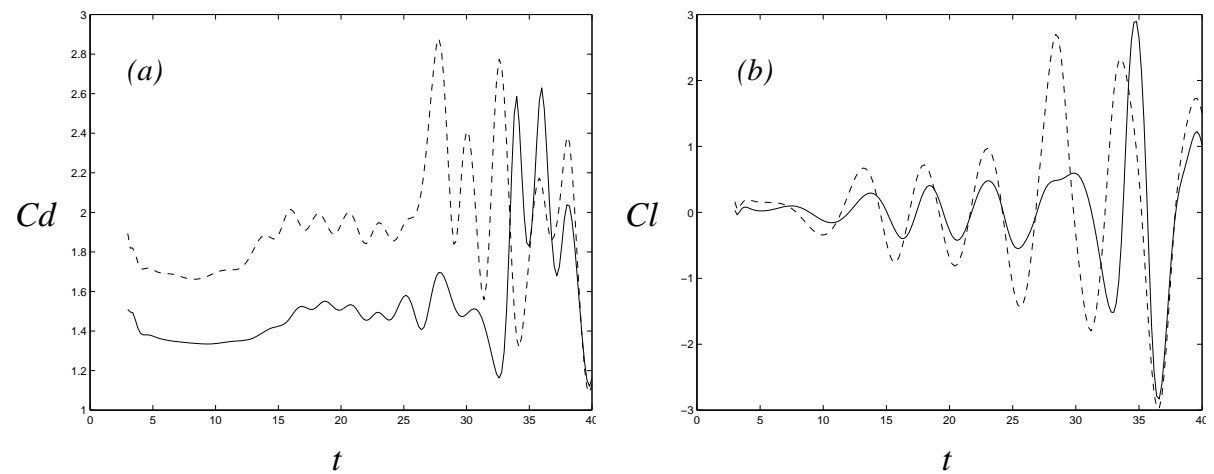

Figure 4: Forces on single cylinder as a function of time: - without smoothing, - - - with smoothing. (a) Drag $C_{d}$. (b) Lift $C_{l}$. Note that smoothing the edge of the cylinder significantly increases the drag and lift, although the vortex shedding frequency is not affected greatly.

particularly easy to implement with the penalization scheme (the mask changes in position and form in response to the flow forces). Note that it is generally believed that the flow in tube bundles remains largely two-dimensional (even at high Reynolds numbers) because of the close packing of the tubes and because cylinder oscillations tend to correlate the fluid motion along the array axis. For this reason a two-dimensional simulation should give useful results in this case.

The cylinder array considered here is a square array where the pitch to diameter ratio is fixed at $P / D=1.5$ (see figure 5). The cylinder is fixed and there is one cylinder per periodic cell (which limits the largest flow structures). Two cases are considered: in-line (mean flow along the axis of the cylinder array) and rotated (mean flow along a diagonal of the array). To apply the mean flow a uniform velocity (i.e. a nonzero $\boldsymbol{k}=0$ Fourier mode) is added at the desired angle. These two cases were chosen because experimental results suggest they produce very different flow regimes [19], and there is some controversy over the precise nature of vortex generation in each regime.

The flow through the two cylinder arrays (in-line and rotated) were calculated at 23 Reynolds numbers (based on cylinder diameter and mean flow velocity) in the range $10 \leq R e \leq 4000$. At each $R e \leq 1000$ the de-aliased resolution was adjusted so that there are at least 4 points across the boundary layer $\left(R e^{-1 / 2} /(3 / 2 \Delta x) \geq 4\right)$ and from $1000<R e \leq 4000$ we ensured the that $R e^{-1 / 2} /(3 / 2 \Delta x) \geq 2$. This criterion was checked using a grid refinement study at low Reynolds number, and ensuring the error in the RMS (root mean square) drag was less than $2.5 \%$. The maximum resolution used was $288 \times 288$ and the minimum resolution was $128 \times 128$. In all simulations we set $\epsilon=10^{-3}$. To save 


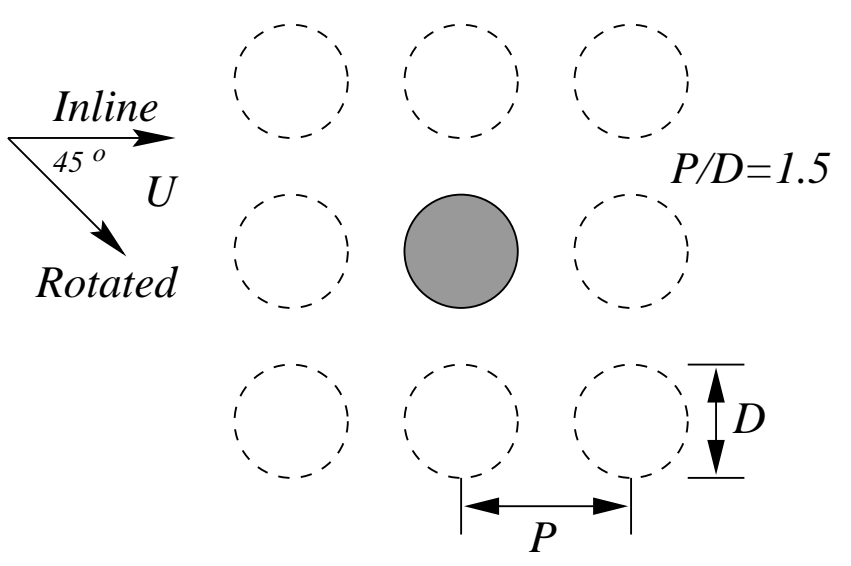

Figure 5: Configuration of periodic cylinder arrays.

computer time the instability was triggered by slightly oscillating the cylinder at the beginning of the simulation. Drag, lift, vorticity and Strouhal number were measured for each flow. Since there are many different shedding frequencies at higher Reynolds numbers, the Strouhal number is defined using the peak of the lift force frequency spectrum.

A typical snap-shot of the flow through the in-line array at $R e=1000$ is shown in figure $6(\mathrm{a})$. The flow has a characteristic jet shear-layer structure, and there is no sign of vortex shedding behind the cylinders. However, it appears that vortices are generated by a shear-layer instability at the edge of the shearlayer between the cylinders. The overall form of the flow and the generation of vorticity via a shear-layer instability is also seen in the laboratory experiment of Ziada \& Oengören [20] reproduced in figure 6(b). In contrast, the flow past the rotated array (see figure 6c) exhibits a completely different flow regime. The flow is characterized by the periodic generation and shedding of vortices from the cylinders, and the region between the cylinders resembles two-dimensional turbulence (with strong vortex-vortex interactions). The production of vorticity in the rotated array is the same as in an isolated cylinder. The vortex shedding observed here is qualitatively similar to that seen in laboratory experiment of Weaver et al. [21] shown in figure 6(d). It is clear that the flow regimes are completely different for the in-line and rotated square arrays, even though the Reynolds numbers are identical. Thus, our numerical results confirm the experimental observations of different mechanisms of vortex generation in inline and rotated square arrays made by Ziada and Weaver. The precise nature of vortex generation mechanisms in cylinder arrays was previously unclear.

The plots of vorticity in the in-line and rotated arrays showed very different 


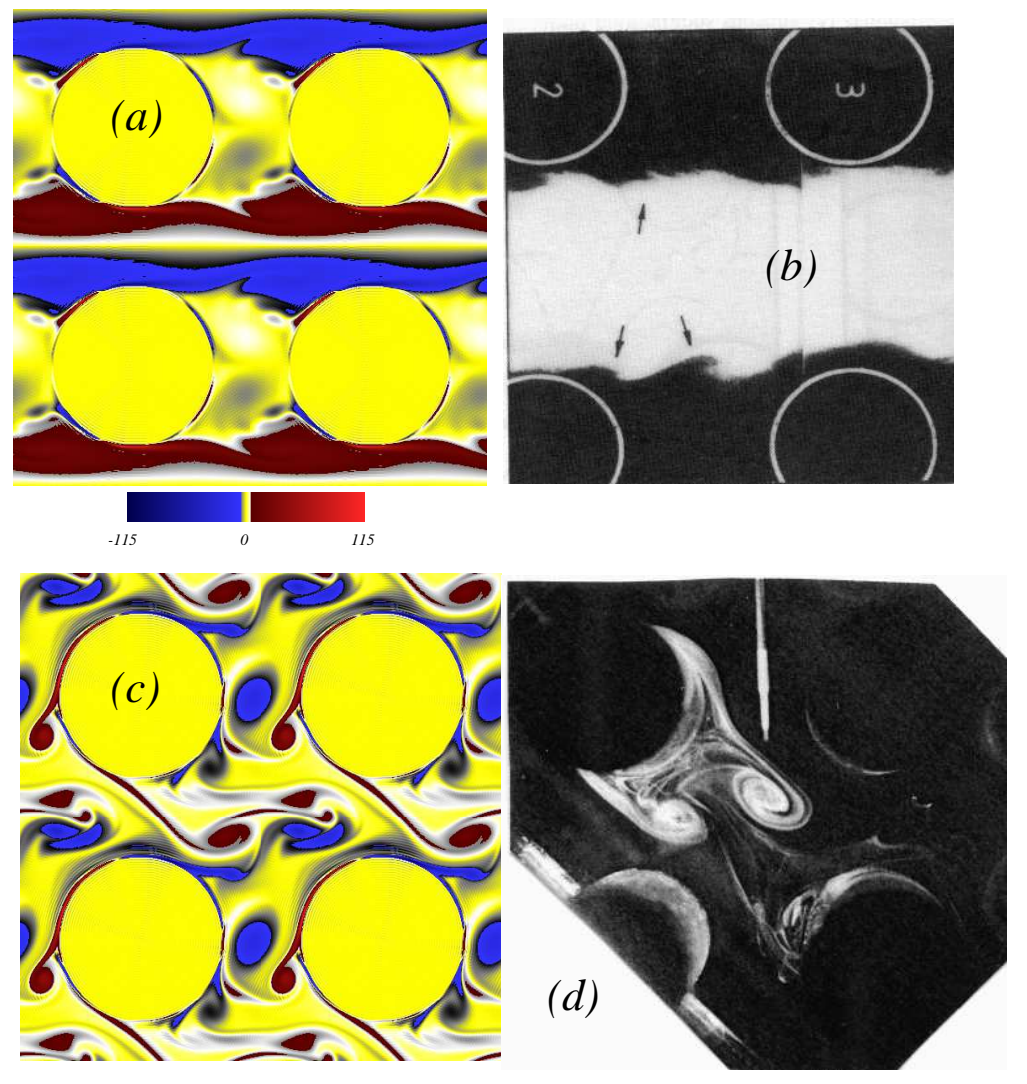

Figure 6: Vorticity in closely-spaced square cylinder arrays (copied four times for clarity). (a) Vorticity in in-line square array at $R e=1000$. Note the presence of jet-like structures and the apparent absence of vortex shedding. Vortices appear to be generated by shear instability along the jet shear layer. (b) Laboratory photograph of jet shear layer in in-line array with $P / D=1.75$ and $R e=730$ [20]. (c) Vorticity in rotated square array (copied four times for clarity) at $R e=1000$. The vorticity structure is completely different from that of the in-line array: many small vortices are produced in the boundary layer and shed downstream. The flow between the cylinders is reminiscent of two-dimensional turbulence. (d) Laboratory photograph of vortex shedding in a rotated square array with $P / D=1.5$ and $R e=967$ from Weaver et al. [21]. The vortex shedding mechanism and vortex scale are similar to that observed in (c). 
mechanisms of vorticity generation at the same Reynolds number. Since vortex shedding is the main source of force on the cylinder one would expect different values of drag, lift, and Strouhal frequency for in-line and rotated arrays. The RMS drag, RMS lift, RMS vorticity (spatially and temporally averaged) and the Strouhal frequency are shown as a function of $R e$ in figure 7 . As expected, the RMS drag is significantly higher in the rotated square array after about $R e=40$. Not much data is available on RMS drag in closely spaced cylinder arrays, but the value 2.3 quoted by Price et al. [22] for an in-line array in the range $1.7 \times 10^{3} \leq R e \leq 10^{5}$ is in reasonable agreement (note that the drag does not appear to depend on Reynolds number in this range). The RMS lift is also higher, and lift develops earlier in the rotated array (at $R e=60$ compared to $R e=100$ ). The plots of vorticity suggest that more vorticity is produced in the rotated array, and this is indeed borne out by figure $7(\mathrm{c})$. The Strouhal frequency as a function of Reynolds number is shown for the in-line and and rotated arrays in figure $7(\mathrm{~d})$. The Strouhal frequency for the rotated array is higher than that of the in-line array until about $R e=500$. The shaded region indicates the range of values found experimentally for different tube rows by Price et al. [22] for a rotated square array. Note that the numerical results are within the experimental range until $R e=500$. This may indicate that the threedimensional effects become important at $R e=500$ (note that this is much later than for an isolated cylinder where three-dimensional effects become important around $R e=180$ ).

In summary, in-line arrays produce vorticity as a shear-layer and vortices are generated by a shear-layer instability, whereas rotated square arrays produce vorticity by vortex shedding as in the case of an isolated cylinder. The unsteady forces on the cylinder are much stronger for the rotated array. The numerical results compare well with available quantitative and qualitative experimental data.

\section{$5 \quad$ Summary and conclusions}

The suitability of simulating flow through an array of solid obstacles at moderate Reynolds number using a pseudo-spectral code with Brinkman penalization has been investigated. The Brinkman penalization was introduced by Angot et al. [7] based on the Brinkman equation for flow through a porous medium. Angot et al [7] have shown analytically that the solution of the penalized equation converges to that of the Navier-Stokes equations with the correct boundary conditions when the penalization parameter $\epsilon \rightarrow 0$. The advantage of penalization methods is that the way of introducing solid boundaries is independent of the numerical method and grid used. To test the limits of this method we applied it to pseudo-spectral simulation of flow through a periodic array of cylinders in two dimensions using the $\omega-\psi$ formulation. This is perhaps the most extreme test of the penalization since the Fourier basis has global support (which means it is 

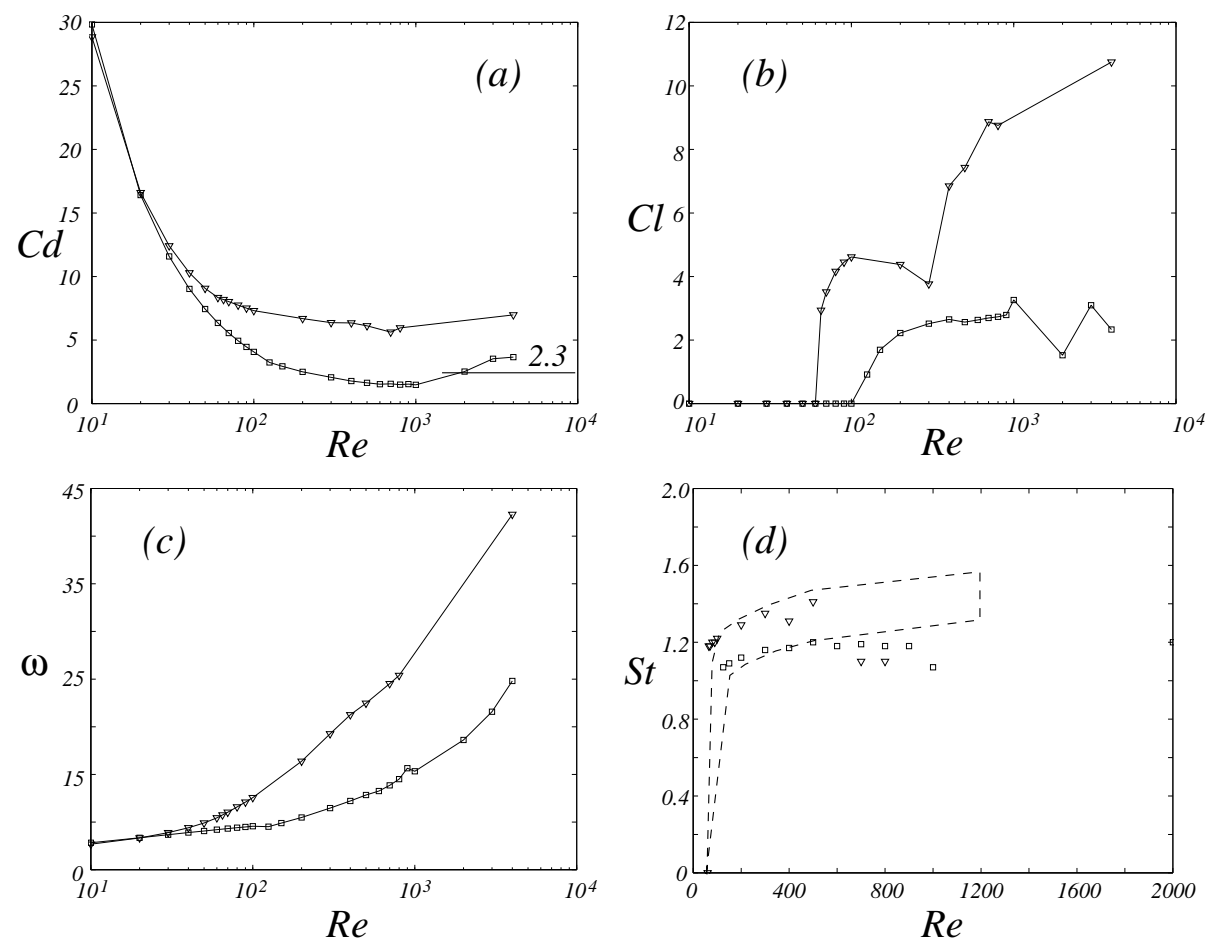

Figure 7: Forces and Strouhal number as a function of Reynolds number for the in-line (squares) and rotated square (triangles) arrays. (a) RMS drag compared with laboratory value stated for the range $1.7 \times 10^{3} \leq R e \leq 10^{5}$ by Price et al. [22]. (b) RMS lift. (c) RMS vorticity $\omega$. (d) Strouhal vortex shedding frequency $S t=f D / U_{\infty}$ compared with laboratory values (region indicated by dotted line) for an rotated square array [22]. 
poorly adapted to non-smooth functions) and the vorticity is a derivative of the velocity (and is therefore less smooth). If the penalization method works reasonably well in this application, then we would expect it to perform acceptably with any numerical method.

To understand analytically the properties of the penalized solution we considered the problem of impulsively started uni-directional flow over a flat plate (Stokes flow). In this case the penalized equations can be solved analytically. It was determined that the velocity is $C^{1}$ at the solid boundary (the velocity and its gradient continuous, but its second derivative is discontinuous). The error in satisfying the boundary condition is $O\left(\epsilon^{1 / 2}\right)$, but the error compared to the exact solution is very small at a finite distance from the boundary. In addition, the velocity inside the solid decreases exponentially away from the boundary (it is evanescent) and is only significant to a skin-depth $O\left(\epsilon^{1 / 2}\right)$. The error in the force on the obstacle is only $O(\epsilon)$ and, surprisingly, the error in the shear stress at the wall is exponentially small, $O(\exp (-t / \epsilon))$. The properties of the solution near the boundary in general three-dimensional flows is expected to be similar. These results suggest that the Brinkman penalization should give very accurate simulations of the flow around obstacles. Numerical solution of the equations for uni-directional channel flow (the periodic equivalent of Stokes flow) showed linear convergence of the $L^{1}$ error with $\Delta x$ for both 2nd-order finite difference and pseudo-spectral methods. However, we observed a form of quadratic 'superconvergence' for the particular relation $\epsilon \approx \Delta x^{2.5--3}$. Although the order of the pseudo-spectral method is in general only linear, the absolute value of the error is quite small ( $10^{-3}$ for 128 points) and there is no sign of Gibbs oscillations.

The penalization method was then applied to a pseudo-spectral simulation of two-dimensional flow through cylinder arrays. This problem was chosen because it is well-adapted to the pseudo-spectral method (due to its periodic boundary conditions) and because it is technologically relevant (to heat exchangers). The physics of periodic cylinder arrays have also not been extensively studied by the fluid mechanics community. The first configuration considered was widely separated cylinders in long domains. This configurations approximates isolated cylinders for moderates times and allows comparison with well-established results. The periodic shedding of vortices at $R e=200$ was observed an the Strouhal number was within $10 \%$ of that observed for a truly isolated cylinder in a laboratory experiment.

The next configuration considered was a closely spaced square array of cylinders with a pitch to diameter ratio of 1.5. This configuration is similar to that of heat exchanger tubes in steam generators, and has been extensively studied by mechanical engineers [19]. We considered the case where the tubes are fixed (they do not deform or move in response to the fluid forces). Two different cases were considered: in-line (mean flow along the array axis) and rotated (mean flow along the array diagonal). Experiments have suggested that these two cases have very different flow regimes. The penalized two-dimensional vorticity-stream function pseudo-spectral code was solved at a Reynolds num- 
bers in the range $10 \leq R e \leq 4000$ for both cases. We confirmed that the in-line and rotated arrays have different flow regimes at the same Reynolds number. In particular, the form of vortex generation is different. In the in-line array vorticity is present in the form of jet shear-layers, and vortices are generated through a shear instability at the inner edge of the shear layer. In the rotated array, however, vortices are generated by periodic shedding off the cylinder boundary (as in the case of an isolated cylinder). Consequently, the rotated array has significantly higher lift and drag at the same Reynolds number and the flow becomes turbulent (i.e. characterized by coherent vortices and vorticity filaments) at a lower Reynolds number. RMS drag for the in-line array agrees well with high Reynolds number experiments, while the Strouhal frequency for the rotated array agrees well up to $R e=500$. These results suggest (as commonly believed) that the forces generated in closely spaced cylinder arrays are largely two-dimensional in origin.

The penalization method allows the obstacles to move or deform over time with no additional complication of the code. This property should allow us to truly model fluid-structure interaction by allowing the cylinders to move or deform in response to fluid forces. This addition to the code is now under investigation.

In the two-dimensional calculations we used a Adams-Bashforth method in time for the nonlinear term, and this enforced the relation $\epsilon \sim \Delta t$. Ideally, $\epsilon$ should be varied independently of $\Delta t$ (that is one of the advantages of the penalization), and the stiffly stable Krylov method used for the one-dimensional flow allows this. We are currently implementing the Krylov method in the twodimensional code.

A major advantage of the pseudo-spectral method is that it is easy to implement in three dimensions. The passage from two to three dimensions is straightforward and will be undertaken soon.

The fact that the penalization method has proved useful, even when applied to such a poorly adapted numerical method, indicates that Brinkman penalization should be useful for a wide variety of flows and numerical methods. In particular, this study is seen as the first step in developing a wavelet-based method for the calculation of high Reynolds number flows in complex geometries. Wavelets have the advantage of compact support (and are thus better suited to functions with strong gradients), and adaptivity in space and scale (one uses a uniform grid, but only calculates wavelet coefficients at the positions and scales that are active). The implementation of the Brinkman penalization to wavelet simulations is currently being studied.

The present investigation has shown the potential of the Brinkman penalization for accurate simulation of flow through complex geometries, and has suggested many directions for further work. The combination of penalization with wavelet methods have the potential to allow high Reynolds number simulation of turbulent flow in actual engineering configurations without turbulence modelling. 


\section{Acknowledgments}

This work is a result of the PPF programme on turbulence modelling of the Ecole Normale Supérieure de Cachan and the Ecole Normale Supérieure de Paris. We thank M. Farge and A. Poitou for bringing together many people interested in CFD, and C. Bénard for her support. We would also like to thank K. Schneider for help with development of the numerical codes used to produce the results in this paper. We would especially like to thank P. Fabrie, P. Angot and C.-H. Bruneau for explaining their penalization method to us. This work has been improved by the suggestions of P. Roe, T. Hurd, J. Boland and L. Tuckermann. N. Kevlahan thanks McMaster University and Ecole Normale Supérieure de Cachan for their financial support. The authors acknowledge financial support from Dassault-Aviation.

\section{References}

[1] C. S. Peskin, "Flow patterns around heart valves: a numerical method," J. Comput. Phys. 10, 252-271 (1972).

[2] D. Goldstein, R. Handler, and L. Sirovich, "Modeling a no-slip flow boundary with an external force field," J. Comput. Phys. 105, 354-366 (1993).

[3] D. Goldstein and T.-C. Tuan, "Secondary flow induced by riblets," J. Fluid Mech. 363, 115-151 (1998).

[4] E. M. Saiki and S. Biringen, "Simulation of boundary layer transition: effects of a spherical particle," J. Fluid Mech. 345, 133-164 (1997).

[5] J. Mohd-Yusof and J. L. Lumley, "Simulations of flow around cylinders using boundary forcing," Bull. of APS (DFD) 39(9), 1861 (1994).

[6] F. Tremblay, M. Manhart and R. Friedrich, "DNS of flow around a circular cylinder at a subcritical Reynolds number with cartesian grids," In Advances in Turbulence VIII, 659-662 (2000).

[7] P. Angot, C.-H. Bruneau, and P. Fabrie, "A penalization method to take into account obstacles in viscous flows," Numerische Mathematik 81, 497520 (1999).

[8] E. Arquis and J. P. Caltagirone, "Sur les conditions hydrodynamiques au voisinage d'une interface milieu fluide - milieu poreux : application à la convection naturelle," C. R. Acad. Sci. Paris II 299, 1-4 (1984).

[9] K. Khadra, S. Parneix, P. Angot, and J.-P. Caltagirone, "Fictitious domain approach for numerical modelling of Navier-Stokes equations," Int. J. Num. Methods in Fluids (accepted) (1999). 
[10] J.P. Caltagirone, "Sur l'interaction fluide-milieu poreux; application au calcul des effort exercés sur un obstacle par un fluide visqueuex," C. R. Acad. Sci. Paris II 318, 571-577 (1994).

[11] P. Angot "Analysis of singular perturbations on the Brinkman problem for fictitious domain models of viscous flows," Math. Meth. Appl. Sci. 22, 1395-1412 (1999).

[12] M. Kac, "On some connections between probability theory and differential and integral equations," In Proc. 2nd Berkeley Symp. Math. Stat. Prob., J. Neyman, ed., pp. 189-215 (Univ. Cal., 1951).

[13] M.-C. Lai and C. S. Peskin, "An immersed boundary method with formal second-order accuracy and reduced numerical viscosity", J. Comput. Phys., 160, 705-719 (2000).

[14] A. Vincent and M. Meneguzzi, "The spatial structure and statistical properties of homogeneous turbulence," J. Fluid Mech. 225, 1-20 (1991).

[15] W. S. Don, "Numerical study of pseudospectral methods in shock wave applications," J. Comp. Phys. 110, 103-111 (1994).

[16] W. S. Edwards, L. S. Tuckerman, R. A. Friesner, and D. C. Sorensen, "Krylov methods for the incompressible Navier-Stokes equations," J. Comp. Phys. 110, 82-102 (1994).

[17] G. K. Batchelor, An Introduction to Fluid Dynamics (Cambridge University Press, 1967).

[18] C. H. K. Williamson, "Oblique and parallel modes of vortex shedding in the wake of a circular cylinder at low Reynolds numbers," J. Fluid Mech. 206, 579-627 (1989).

[19] D. S. Weaver, "Vortex shedding and acoustic resonance in heat exchanger tube arrays," In Technology for the '90s, M. K. Au-Yang, ed., pp. 776-810 (ASME, 1993).

[20] S. Ziada and A. Oengören, "Vorticity shedding and acoustic resonance in an in-line tube bundle. Part I: vorticity shedding," J. Fluids and Structures 6, 271-292 (1992).

[21] D. S. Weaver, H. Y. Lian, and X. Y. Huang, "Vortex shedding in rotated square tube arrays," J. Fluids Structures 7, 107-121 (1993).

[22] S. J. Price, M. P. Païdoussis, and B. Mark, "Flow visualization of the interstitial cross-flow through parallel trangular and rotated square arrays of cylinders," J. Sound and Vibration 181, 85-98 (1995). 